\title{
Laparoscopy assisted ERCP
}

\begin{abstract}
With the pandemic of obesity, bariatric surgery in gaining popularity. Bariatric surgery increases the risk of gall stone disease. This is a case report of a young woman who presented with symptomatic choledocholithiasis and cholelithiasis. She has previous Rouxen-Y bariatric surgery for obesity 4 years ago which made standard Endoscopic Retrograde Cholangiography (ERC) not feasible. Therefore, ERC was attempted using a conventional colonoscope but was unsuccessful. She later underwent Laparoscopy and ERC via gastric stoma followed by subsequent cholecystectomy. There was close collaboration between the surgeon and the endoscopist to harmonize their timetable for the combined procedure. The aim of this paper is to report this case of laparoscopically assisted ERCP (LA-ERCP) combined with cholecystectomy, technical challenges and highlight the difficulties encountered when dealing with choledocholithiasis in such patients who have undergone previous bariatic surgery.
\end{abstract}

Volume 7 Issue 2 - 2017

\author{
Zaki Alhashimalsayed,' Nassir Al Hayaf, ${ }^{2}$ \\ Diamond Joy ${ }^{2,3}$ \\ 'Department of Internal Medicine, King Fahad Specialist \\ Hospital, Saudi Arabia \\ ${ }^{2}$ Department of Gastroenterology, Johns Hopkins Aramco \\ Hospital, Saudi Arabia \\ ${ }^{3}$ Department of Gastroenterology, The James Cook University \\ Hospital, United Kingdom
}

Correspondence: Diamond Joy, Room A-428-9, Building 6I, Dhahran Health Center, Johns Hopkins Aramco Hospital, Saudi Aramco, Dhahran 3131 I, Saudi Arabia, Tel +966- I3-877-9748, Fax 966-13-877-3790, Email diamond.joy@JHAH.com

Received: June II, 2017 | Published: July 24, 2017

\section{Introduction}

Obesity is associated with adverse health outcomes including cardiovascular diseases and cancer. A recent study looking at obesity trend suggests that by 2025 , the prevalence of obesity worldwide will reach $18 \%$ for males and surpass $21 \%$ in women. ${ }^{1}$ The trend of obesity in the Middle East is particularly pronounced and exceeds 50\% among women in many countries in this region. Bariatric surgery has been found to be effective for weight loss and reduce the consequences of obesity.

However, long term health consequences of bariatric surgery are not fully clear. The weight loss that accompanies bariatric Roux-en-Y gastric bypass (R-e-YGB) is a risk factor for cholelithiasis which in turn increases the risk of choledocholithiasis and gall stone pancreatitis. Around $7-8 \%$ of post R-e-YGB patients become symptomatic with gall stone disease that require cholecystectomy. Therefore, due to this relative low prevalence rate of gall stone disease, recommending prophylactic cholecystectomy in patients undergoing R-e-YGB might be an overkill and is not generally advocated. ${ }^{2}$ While performing cholecystectomy in R-e-YGB patients is relatively straightforward, performing Endoscopic Retrograde Cholangiopancreatography (ERCP) with conventional scope is technically challenging in these cases.

We report a case of laparoscopically-assisted ERCP with review of the literature.

\section{Case report}

A 30-year-old woman status post R-e-YGB surgery for obesity 4 years ago. She was admitted with acute gall stone pancreatitis. Biliary imaging confirmed choledocholithiasis and cholelithiasis. ERCP was attempted using a conventional colonoscope under conscious sedation using midazolam and fentanyl. However, despite entering the pancreatico-biliary limb (roux limb) of the intestine and visualizing bile, this was not successful in identifying the ampulla. This procedure took around 2 hours and had to be abandoned.

She later underwent Laparoscopy with assisted ERCP and subsequent cholecystectomy. The patient had standard laparoscopy for access into the peritoneal cavity. After identification of the gastric remnant, the therapeutic duodenoscope was introduced into the abdominal cavity via left upper quadrant incision and inserted into the remnant stomach through the gastrostomy. It was passed into the duodenum with access to the ampulla without difficulties. The common bile duct was cannulated using guide wire. Cholangiogram reveled stones in the common bile duct which were extracted after sphincterotomy with balloon. It was an uncomplicated procedure. The total operative time was 100 minutes. The patient was discharged the next day with good recovery. The patient was reviewed a month later and was asymptomatic.

There were no real difficulties in manipulating the duodenoscope. There were no ERCP related complications. The postoperative care was similar to conventional cholecystectomy or other laparoscopy procedures. There was close collaboration between the surgeon and the endoscopist to harmonize their timetable and to ensure optimal positioning of the endoscopic and radiological equipment during the procedure. The patient remained well and was asymptomatic for the next one year of follow up (Figure 1).

\section{Discussion}

With the pandemic proportion of obesity related surgeries being performed, it is only natural to expect increasing frequency of RYGB surgeries. Performing the conventional colonoscopy assisted ERCP in the patient had to be abandoned because of the challenges faced during the procedure. These challenges may include the followings:

i. First: the altered anatomy makes accessing the ampulla very difficult. Although there are newer scopes, the significant distance to the ampulla in the pancreatico-duodenal limb makes the procedure difficult [1]. Often due to the lengths involved, colonoscopies are used for the procedure.

ii. Second: The ampulla is in an upside-down orientation, which makes visualization difficult especially with a front-viewing endoscope. Furthermore, these scopes do not come with elevators, which makes maneuvering to an optimal position particularly difficult. 
iii. Third: The accessories required to assist the long scopes required for ERCP are very few. For example, it is not easy to obtain a long sphincterotome or balloon that will pass through the colonoscope with adequate working length.

iv. Fourth: Long bypass limp and a prevent access to the major ampulla. It has been reported that there is a significantly higher failure rate if the total bypass limp length is greater than $150 \mathrm{~cm} .{ }^{1}$

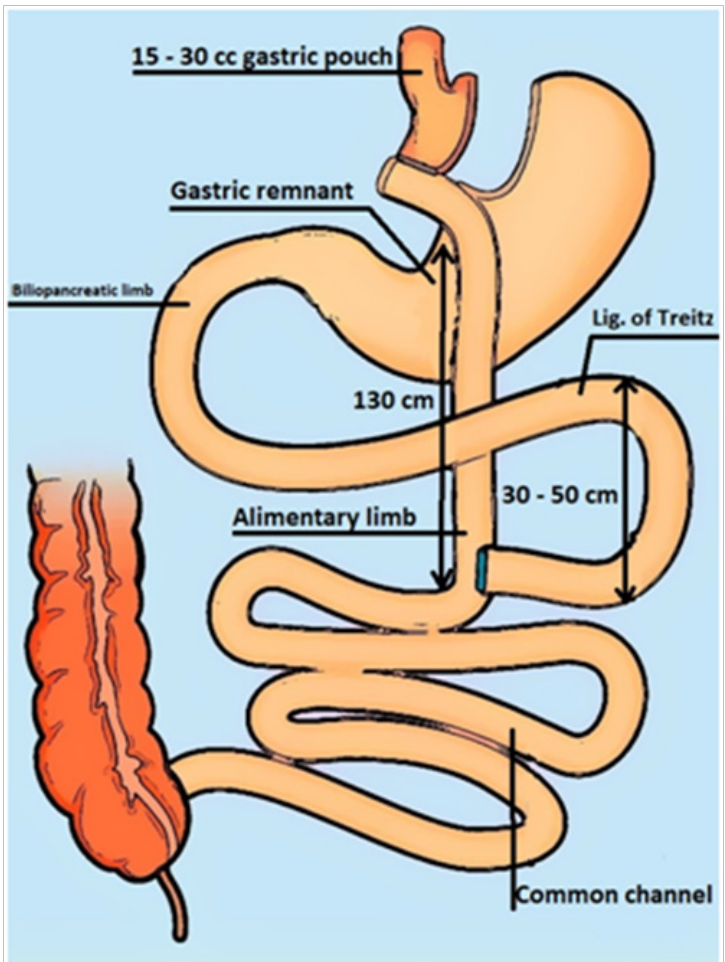

Figure I Roux-en-Y gastric bypass. Side-to-side jejunojejunostomy is performed after dividing proximal jejunum distal to the ligament of Treitz. The gastric pouch is created and oriented vertically with exclusion of the gastric fundus. The Roux limb is brought up in a retro colic orientation anterior to the stomach remnant and a gastrojejunostomy is performed. The Roux limb is usually $\pm 130 \mathrm{~cm}$ and the biliopancreatic limb from the ligament of Treitz to the jejuno-jejunal anastomosis commonly varies from 30 to $50 \mathrm{~cm}$.

Laparoscopic assisted ERCP and combined cholecystectomy as a first-line therapy helps avoid the need for further procedure; has equivalent morbidity and hospital stay as cholecystectomy. Furthermore, this preempts the need for the technically and temporally demanding prior colonoscopic ERCP procedure. LA-ERCP has a high success rate of over $95 \%$. In contrast, ERCP using colonoscope or balloon enteroscope can be time consuming, requires high skill level and the success rate of biliary cannulation around $60 \%$ in most of the literature..$^{2-6}$

Although in this case, the duodenoscope was introduced directly via the laparoscopic opening into the stomach; it is worth considering placing a sterilized overtube into the stomach. This will help guide the scope and reduce the risk of contamination of the peritoneal cavity. Another advantage of the overtube is that it could offers stability for positioning of the scope. Other techniques have been used to obtain biliary access after Roux-en-Y gastric bypass surgery. This includes minimally invasive techniques like endoscopic ultrasound-guided and fluoroscopy assisted insertion of a gastrostomy tube into the gastric remnant. Following this ERCP is performed percutaneously. ERCP can also be performed using a balloon enteroscope. The success rate of ERCP using balloon enteroscope can be higher and approaches $85 \%$ if the length of the Roux plus the pancreaticobiliary limb is less than $150 \mathrm{~cm} .{ }^{2}$ The main advantage of using the endoscopic approach is the cost advantage. The ampulla is approached from a different angle when using the duodenoscope which results in a different and more cumbersome approach for biliary cannulation. The enteroscope also lacks an elevator which means that maintaining the position of scope is difficult. Either way these techniques are quite time consuming and needs to be followed by cholecystectomy.

The advantages of LA-ERCP include the use of standard ERCP scope with standard accessories and can be performed by those with standard ERCP skills. Furthermore, the endoscopist can reach the ampulla in a few minutes after the surgeon gains access to the gastric remnant. While cost is a significant disadvantage, surgery would still be the first choice in community hospitals where there are limited facilities for balloon endoscopy or where there is time and skill constraints. Furthermore, sometimes the previous operative report of the RYGB surgery might not detail the technique used, postoperative complication or the anatomy and length of the roux as well as pancreatico-biliary limb. ${ }^{7-10}$ The disadvantages of surgery-assisted ERCP are that it involves surgery and that it may take time for the surgeon to identify the gastric remnant due to the altered anatomy from previous surgery in these patients. ${ }^{11-13}$

Table 1 summarize the advantages and disadvantages of LAERCP. There is suggestion in the literature that performing LA-ERCP using assistance of a sterile rigid sigmoidoscope into the antrum for use as an overtube helps to stabilize the scope and reduce valuable operative time to less than 100 minutes. $^{2}$ With the improvement in experience and technique LA-ERCP has been found to take less time of around 94 minutes. $^{2}$

Table I This table summarize the advantages and disadvantages of using laparoscopic assisted ERCP

\begin{tabular}{ll}
\hline Advantages & Disadvantages \\
\hline Cost effective & $\begin{array}{l}\text { Expensive, but cheaper than separate complex time consuming ERCP and } \\
\text { separate cholecystectomy. }\end{array}$ \\
Significantly shorted endoscopy procedure time & $\begin{array}{l}\text { Total procedure time might be increased. } \\
\text { Difficult subsequent cholecystectomy due intestinal distension caused by } \\
\text { ERCP }\end{array}$ \\
Standard Side viewing scope can be used & $\begin{array}{l}\text { Difficult to manage subsequent complications like bleeding by endoscopy. } \\
\text { Increased risk of post-ERCP pancreatitis in patients with sphincter of Oddi } \\
\text { dysfunction or papillary stenosis. } \\
\text { Carbon dioxide gas insufflation is advised which is safer than conventional } \\
\text { High biliary cannulation rates, nearly } 100 \%\end{array}$
\end{tabular}

Postoperative care is like conventional laparoscopic cholecystectomy. 


\section{Conclusion}

The prevalence of altered upper gastrointestinal anatomy is not uncommon with increasing obesity and popularity of RYGBP. Laparoscopy-assisted ERCP is a direct approach in the treatment of Roux-en-Y gastric bypass patients to ensure biliary clearance. It is safe and allows endoscopic treatment and cholecystectomy to be performed in a single setting. Combining LA-ERCP and cholecystectomy as a first-line approach is a valid treatment strategy to reduce total cost because the two procedures are performed in the same intervention.

\section{Acknowledgments}

None.

\section{Conflicts of interest}

The authors declare there are no conflicts of interest related to the article.

\section{Funding}

None.

\section{References}

1. NCD Risk factor collaboration. Trends in adult body-mass index in 200 countries from 1975 to 2014: a pooled analysis of 1698 populationbased measurement studies with $19 \cdot 2$ million participants. Lancet. 2016;387(10026):1377-1396.

2. D’Hondt M, Sergeant G, Deylgat B, et al. Prophylactic cholecystectomy, a mandatory step in morbidly obese patients undergoing laparoscopic Roux-en-Y gastric bypass. J Gastrointest Surg. 2011;15(9):1532-1536.

3. Khashab MA, Patrick I Okolo III. Accessing the pancreatobiliary limb and ERCP in the bariatric patient. Gastrointest Endoscopy Clin N Am. 2011;21(2):305-313.
4. Schreiner MA, Chang L, Gluck M, et al. Laparoscopy-assisted versus balloon enteroscopy-assisted ERCP in bariatric post-Roux-en-Y gastric bypass patients. Gastrointest Endosc. 2012;75(4):748-756.

5. Christophe Snauwaert, Pierre Laukens, Bruno Dillemans, et al. Laparoscopy-assisted transgastric endoscopic retrograde cholangiopancreatography in bariatric Roux-en-Y gastric bypass patients. Endosc Int Open. 2015;3(5):E458-E463.

6. Schreiner MA, Chang L, Gluck M, et al. Laparoscopy-assisted versus balloon enteroscopy-assisted ERCP in bariatric post-Roux-en-Y gastric bypass patients. Gastrointest Endosc. 2012;75(4):748-756.

7. Aabakken L. Endoscopic retrograde cholangiopancreatography. Gastrointest Endosc. 2012;76(3):516-520.

8. Bertin PM, Singh K, Arregui ME. Laparoscopic transgastric endoscopic retrograde cholangiopancreatography (ERCP) after gastric bypass: case series and a description of technique. Surgical Endoscopy. 2011;25(8):2592-2596.

9. Emmett DS, Mallat DB. Double-balloon ERCP in patients who have undergone Roux-en-Y surgery: a case series. Gastrointest Endosc. 2007;66(5):1038-1041.

10. Moreels TG. Altered anatomy: enteroscopy and ERCP procedure. Best Pract Res Clin Gastroenterol. 2012;26(3):347-357.

11. Saleem A, Levy MJ, Petersen BT, et al. Laparoscopic-assisted ERCP in Roux-en-Y gastric bypass (RYGB) surgery patients. $J$ Gastrointest Surg. 2012;16(1):203-208.

12. LE Coleman, L Chang Society of American Gastrointestinal and Endoscopic Surgeons.

13. Bharat Paranandi, Deepak Joshi, Borzoueh Mohammadi, et al. Laparoscopy-assisted ERCP (LA-ERCP) following bariatric gastric bypass surgery: initial experience of a single UK centre. Frontline Gastroenterology. 2015. 